\title{
NUMERICAL AND ANALYTICAL CALCULATIONS OF THE FREE SURFACE FLOW BETWEEN TWO SEMI-INFINITE STRAIGHTS
}

\author{
Fatma Zohra Chedala, Abdelkader Amara, Mabrouk Meflah \\ Department of Mathematics, Laboratory of Applied Mathematics, University of Kasdi Merbah \\ Ouargla 30000, Algeria \\ chedalafz@gmail.com,amara.abdelkader@univ-ouargla.dz,meflah.mabrouk@univ-ouargla.dz
}

Received: 29 February 2020; Accepted: 8 November 2020

\begin{abstract}
The problem of two-dimensional flow with the free surface of the jet in a region between two semi-infinite straights intersections at point $O$ is calculated analytically for each angle Beta and numerically for each of the various values of the Weber number and angle Beta. By assuming that the flow is potential, irrotational and that the fluid is incompressible and inviscid, and by taking account only the surface tension for a numerical method using the series truncation, and without the effect of gravity and surface tension for the analytic method utilize the hodograph transformation. The obtained results confirmed a good agreement between them when the Weber number tends to infinity, and the comparison of these surface shapes is illustrated.
\end{abstract}

MSC 2010: 30C30, 65E05, 76B07, 76D05, 76D45

Keywords: free surface, flow, weber number, surface tension, free streamline theory, exact solution, series truncation

\section{Introduction}

Free-surface problems occur in many aspects of science and engineering. They can be defined as problems whose mathematical formulation involves surfaces that have to be found as part of the solution; for some recent development on the topic (see [1-6]) and the references therein. In this literature, we show some contributions of researchers to the finding of a mathematical solution for free-surface problems. The flow sketched in Figure 1 was solved numerically by Vanden-Broeck and Joseph B. Keller [7]. Considering the effect of gravity and neglecting the surface tension, they computed the problem for different values of the Froude number.

Merzougui et al. [8] studied the two-dimensional potential flow in a channel contra an inclined wall. The problem is solved numerically for various values of the Weber number $\alpha$ where the effect of gravity is neglected.

In [9] and [10], the authors find the exact solution for $\beta=0$ and $\beta=\frac{\pi}{2}$ respectively, where the effect of surface tension and effect of gravity are neglected. 
Our assumed problem will be more general than the problems considered before and aforementioned above. In this study, we consider a two-dimensional free surface potential flow of a jet in a region between two straight, semi-infinite intersections that make an angle $\beta$ (Fig. 1). By assuming that the fluid is invicid, incompressible and irrotational in flow, we take into account the effects of surface tension but we neglect all the other forces.

Without the effects of surface tension and the gravity, we can find the exact solution. When we suppose that the gravity or the effects of surface tension exists, the nonlinear boundary condition on a free surface described as a Bernoulli's equation, getting the analytic solution is very difficult, which leads us to search for an approach solution by using a Series Truncation Technique (see e.g.: [7,11-22]). In our research and with the same technique, we are going to treat our problem firstly in the case when the effect of the surface tension is taken into account and when we neglected the effect of gravity. Bernoulli's equation is reformuled by a dimonsionless parameter called Weber number $\alpha$ and the angle $\beta$. The Weber number is a dimensionless characteristic number used to describe fluid flows where there is an interface between two different fluids. In our case we have the liquid and the atmosphere. As a dimensionless quantity, it describes the surface tension force acting on a fluid surface element due to the difference between the fluid pressure and the atmospheric pressure. Its influence is on the shape of the free surface and exactly in the curvature of the surface. This number defined by:

$$
\alpha=\frac{\rho U^{2} H}{T}
$$

where $T$ is the surface tension and $\rho$ is the density of the fluid.

We obtain numerical results, for each value of the angle $\beta$ (for exemple $\beta=\frac{\pi}{4}$ and $\beta=\frac{\pi}{6}$ ) and for various values of Weber number, this leads to a nonlinear algebraic equation system which is solved by Newton's method.

In the second part, and by using the method of the free streamline theory based on the hodograph method and the Schwarz-Christoffel transformation (see, for example, [16]), we found a solution more exhaustive than for all of angle $\beta$.

For the paper, the structure is as follows: A mathematical formulation of the problem is presented in the next section, the numerical procedure is described in Section 3. Section 4 gives the exact solution, and we conclude this work by presenting both the numerical results and discussion in Section 5.

\section{Formulation of the problem}

Consider the move of a two dimensional flow of a fluid due to a jet between two straight semi-infinite intersections at the point $O$. The fluid is assumed to be inviscid, incompressible and irrotational where the effects of gravity are neglected and the 
surface tension is taken into account. In the physical plan $z=x+i y$, one takes $A O$ on the $x o x$. We choose Cartesian coordinates with origin at $O$, and one supposes that the flow is uniform velocity $U$ and of amplitude $\mathrm{H}$, the flow is highly limited by the free thread of current $A^{\prime} M B^{\prime}$.

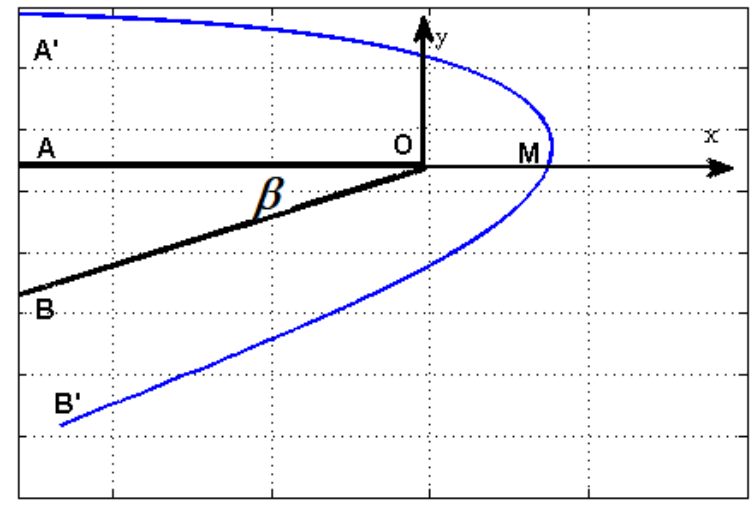

Fig. 1. $z$-plan: sketch of the flow and of the system of coordinates

We consider the flow in the complex plan $z=x+i y$. We are interested in potential flows, and we can define a complex potential function as:

$$
f=\phi+i \psi
$$

where $\phi$ is the potential function and $\psi$ is the stream function. Without loss of generality, we choose $\phi=0$ at $O$ and $\psi=0$ on the streamline $A O B$. It follows from the choice of the dimensionless variables that $\psi=1$ on the free stream line $A^{\prime} B^{\prime}$.

The flow region in the $z$ plan will be mapped via the potential function $f$ onto the semi-infinite strip $(-\infty \leq \phi \leq+\infty, 0 \leq \psi \leq 1)$ (Fig. 2).

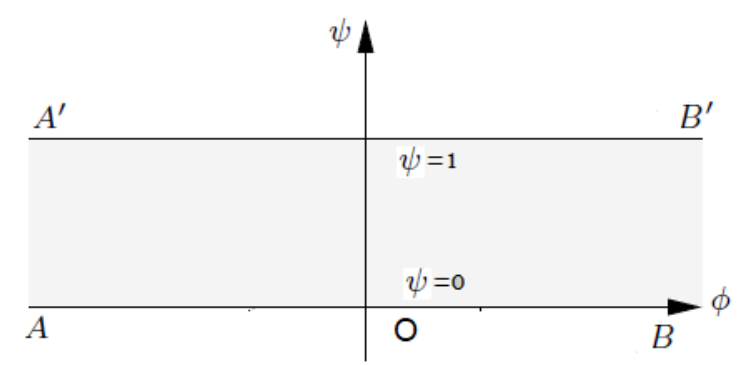

Fig. 2. $f$-plan: The image of the flow in the complex potential plan $f=\phi+i \psi$

The mathematical problem is the satisfied Laplace's equation:

$$
\Delta \phi=0, \quad \text { in the interior of the flow filed, }
$$


the equation of Bernoulli:

$$
\frac{1}{2} q^{2}-\frac{1}{\alpha} K=\frac{1}{2} \quad \text { on } A^{\prime} B^{\prime},
$$

where $\alpha$ is the Weber number, $q$ is the module of the velocity, and $K$ is the curvature of the free surface, and the conditions:

$$
\begin{aligned}
& \frac{\partial \phi}{\partial y}=0, \quad \text { on the horizontal wall } A O \\
& \frac{\partial \phi}{\partial \vec{n}}=0, \quad \text { on the wall } O B
\end{aligned}
$$

where $\vec{n}$ is the unit normal vector of the boundaries.

\section{The numerical solution}

In this section, we study the problem posed in the previous section by taking into account the effect of surface tension, and a numerical method is then adopted. The solution is characterized by the Weber number and the angle $\beta$ by using the series truncation method, which consists in discretizing only the free surface. By using this method, it was possible to accurately determine the singularity nature of the velocity vector at the points of contact.

We use the transformation below:

$$
f=\frac{1}{\pi} \log \left(\frac{1+t}{1-t}\right)^{2}
$$

By this transformation, the band (Fig. 2) is transformed into a upper half unit disk in the plan of variable $t$ (Fig. 3).

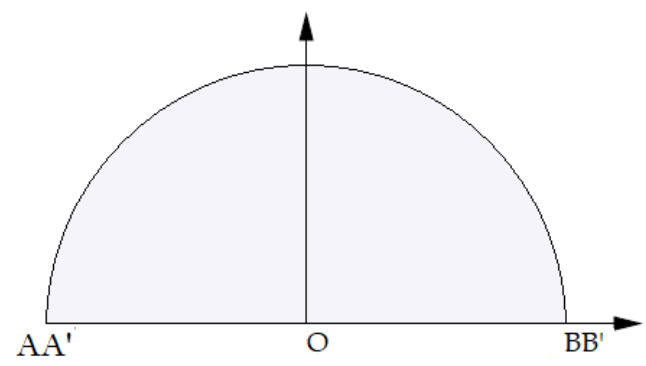

Fig. 3. The image of the flow of Figure 1 in the complex $t$-plan is the upper half unit disk

The free surface $A^{\prime} B^{\prime}$ is transformed on the circumference and the rigid walls on the rays of the disc. The head $O$ of the triangle is placed at the origin. The points $A$, $O$ and $B$ were transformed into $t=-1, t=0$ and $t=1$ at each point. 
All the points of the free surface for the plan $t$ as shown in the relation:

$$
t=|t| e^{i \sigma}=e^{i \sigma}, 0 \leq \sigma \leq \pi
$$

and in the plan $f$ by the relation:

$$
f=\phi+i, \quad \text { such as } \quad-\infty \leq \phi \leq+\infty
$$

We note the velocity vector $\xi(t)$ by:

$$
\xi(t)=u-i v=\frac{d f}{d z}
$$

as $u-i v$ is analytical in the field of flow, $\xi(t)$ can be written as:

$$
\xi=e^{\tau-i \theta}
$$

where $\theta$ is the angle between the velocity vector and the horizontal. In these new variables, Bernoulli's equation (3) becomes:

$$
e^{2 \tau}-\frac{2}{\alpha}\left|\frac{\partial \theta}{\partial \phi}\right| e^{\tau}=1 \quad \text { on } \quad A^{\prime} B^{\prime}, \psi=1,-\infty \leq \phi \leq+\infty
$$

and the conditions (4) and (5) become:

$$
\begin{aligned}
& \operatorname{Im} \xi=0 \quad \text { on } \quad \psi=0,-\infty \leq \phi \leq \phi_{O} \\
& \frac{\operatorname{Re} \xi}{\operatorname{Im} \xi}=\beta \quad \text { on } \quad \psi=0, \quad \phi_{O} \leq \phi \leq+\infty
\end{aligned}
$$

The aim of this part is to find the one function $\tau-i \theta$ that is an analytical in the strip $0<\psi<1$ and to verify the conditions (9), (10) and (11). Since $\xi(t)$ is analytical in all the flow domain except at point $O$, an asymptotic study is necessary to obtain the behaviour of the velocity of this point, we have:

$$
\xi \sim \bigcirc(t)^{1-\frac{\gamma}{\pi}} \quad \text { as } t \longrightarrow 0
$$

Now, let us define the function $\Omega(t)$ as follows:

$$
\xi=g(t) \exp \Omega(t)
$$

which $g(t)$ is composed of singularities and zeros of $\xi$, and $\Omega(t)$ is an analytical function inside the disk unit $|t|<1$, we seek $\xi(t)$ as a series of the form

$$
\xi=u-i v=(t)^{1-\frac{\gamma}{\pi}} \times \exp \left(\sum_{k=0}^{\infty} a_{k} t^{2 k}\right)
$$

when the $a_{k}$ are real constants to be determined. The function (14) verifies the boundary conditions except Bernoulli's condition. The coefficients $a_{k}$ are calculated for Bernoulli's equation to be verified. 
The final form of Bernoulli's equation that is needed for the numerical calculation is given by:

$$
e^{2 \bar{\tau}}-\frac{2}{\alpha}\left|\frac{\partial \bar{\theta}}{\partial \phi}\right| e^{\bar{\tau}}=1
$$

here $\bar{\tau}(\sigma)$ and $\bar{\theta}(\sigma)$ denote the values of $\tau$ and $\theta$ on the free surface $A^{\prime} M B^{\prime}$.

For the determination of the coefficients $a_{k}$, we make a truncation of the series after $N$ terms. In this way, the discretization of the interval $[0, \pi]$ is introduced at $N+1$ points.

$$
\sigma_{I}=-\frac{\pi}{N}\left(I-\frac{1}{4}\right), I=1,2,3, \ldots, N
$$

We use the two equations (15) and (16), we obtain: $[\bar{\tau}(\sigma)]_{\sigma=\sigma_{I}},[\bar{\theta}(\sigma)]_{\sigma=\sigma_{I}}$ and $\left[\frac{\partial \bar{\theta}(\sigma)}{\partial \sigma}\right]_{\sigma=\sigma_{I}}$ are expressed in terms of coefficients $a_{k}$. We get the system with $N+1$ equations in $N+1$ unknowns.

The system obtained is solved by using the Newton method.

The form of the free surface is achieved by numerically integrating the relation:

$$
\left\{\begin{array}{l}
\frac{\partial x}{\partial \sigma}=\exp (-\bar{\tau}(\sigma)) \cos (\bar{\theta}(\sigma)) \frac{\partial \phi}{\partial \sigma} \\
\frac{\partial y}{\partial \sigma}=\exp (-\bar{\tau}(\sigma)) \sin (\bar{\theta}(\sigma)) \frac{\partial \phi}{\partial \sigma}
\end{array}\right.
$$

The numerical scheme described above was used to calculate the approximate solutions for different values of Weber number $\alpha$ as well as the angle $\beta$.

\section{The exact solution}

The objective in this Section is to find the analytical solution of the problem defined in Section 2. In the absence of gravity and surface tension, we can obtain the exact solution of this problem by applying the hodograph method according to the free streamline theory and conformal transformation. We can introduce the complex function $\Omega$ defined by:

$$
\Omega=\log \left(\frac{U}{d f / d z}\right)=\log \left(\frac{U}{u-i v}\right)=\log \left(\frac{U}{q}\right)+i \theta
$$

where $\theta$ is the angle between the velocity vector and the $x$-axis, $q$ is the module speed and $U$ is the reference velocity that can be considered as a unit of velocity.

By this last transformation, the field occupied by the fluid in the z-plan is transformed into an infinite band in the $\Omega$-plan as the shape that is shown in Figure 4. 


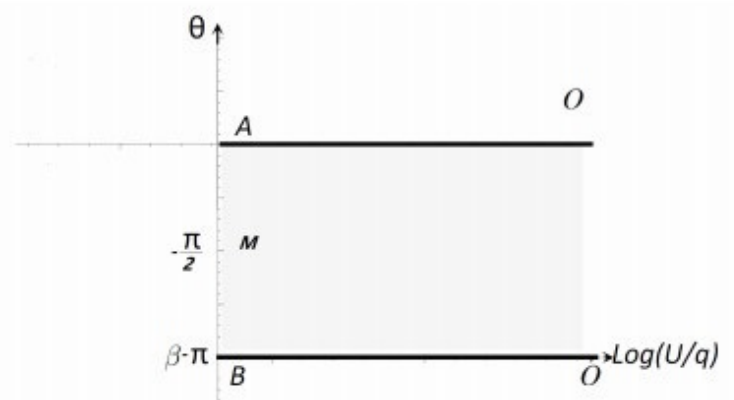

Fig. 4. $\Omega$-plan: Flow configuration in a semi-infinite band

To transform a semi-infinite band from the $\Omega$-plan to the upper half-plan of another complex $\lambda$-plan, we use the conform transformation given by Schwartz-Christoffel's theorem, respecting the direction and orientation of the flow (Fig. 5). This transformation is given by:

$$
\Omega=\left(\frac{\beta}{\pi}-1\right) \log \left(\sqrt{\lambda^{2}-1}+\lambda\right)
$$

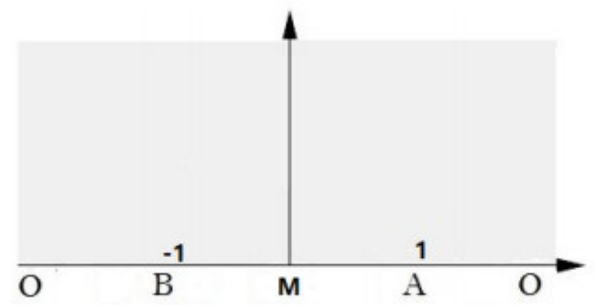

Fig. 5. $\lambda$-plan: Flow configuration in a half plan

On the other hand, the transformation that transforms the interior of the infinite band from the $f$-plan to the upper half plan of the $\lambda$-plan is as follows:

$$
\lambda=\frac{1+e^{\frac{\pi f}{H U}}}{1-e^{\frac{\pi f}{H U}}}
$$

Using the relation: $\frac{d z}{d \lambda}=\frac{d z}{d f} \frac{d f}{d \lambda}$ and after calculations, we obtain:

$$
\frac{d z}{d \lambda}=\frac{2 H}{\pi} \times \frac{\left(\lambda+i \sqrt{1-\lambda^{2}}\right)^{\left(-1+\frac{\beta}{\pi}\right)}}{\lambda^{2}-1}
$$

where $-1 \leq \lambda \leq 1$.

After integration of (20), with the choice of $z_{0}=x_{0}$ into the point $M$. The exact solution of the free surface is as follows: 
For example $\beta=\frac{\pi}{6}$

$$
\left\{\begin{array}{l}
x=x_{0}+H\left(1+\sqrt{3}-\frac{\sqrt{3}}{\pi} \log \frac{2 \cos (C)+\sqrt{3}}{2 \cos (C)-\sqrt{3}}+\frac{1}{\pi} \log \frac{(2 \cos (C)-1)(1-\cos (C))}{(2 \cos (C)+1)(\cos (C)+1)}\right) \\
y=H\left(1-\frac{\sqrt{3}}{\pi} \log \frac{2 \sin (C)+\sqrt{3}}{\sqrt{3}-2 \sin (C)}-\frac{1}{\pi} \log \frac{(2 \sin (C)+1)(\sin (C)+1)}{(2 \sin (C)-1)(1-\sin (C))}\right)
\end{array}\right.
$$

with $C=\frac{\arccos (\lambda)}{6}$. And for $\beta=\frac{\pi}{4}$ we obtain:

$$
\left\{\begin{array}{l}
x=x_{0}+H\left(-1-\sqrt{2}+\frac{\sqrt{2}}{\pi} \log \frac{2 \cos (D)-\sqrt{2}}{2 \cos (D)+\sqrt{2}}+\frac{1}{\pi} \log \frac{1-\cos (D)}{\cos (D)+1}\right) \\
y=H\left(1+\frac{\sqrt{2}}{\pi} \log \frac{\sqrt{2}-2 \sin (D)}{2 \sin (D)+\sqrt{2}}+\frac{1}{\pi} \log \frac{1-\sin (D)}{\sin (D)+1}\right)
\end{array}\right.
$$

with $D=\frac{\arccos (\lambda)}{4}$.

\section{Discussion of results}

\subsection{The flow with surface tension effect}

The numerical method outlined in section 3 is used to calculate solutions for different values of the Weber number and several values of the angle $\beta$. If the influence of surface tension is taken into account, the computational results show that there is a solution for different values $\alpha>15$.

As a consequence, the coefficients $a_{k}$ of series (14) were found to decrease very quickly for different values of $\beta$ and Weber number, for example, when $\beta=\frac{\pi}{6}$ and for different values of the Weber number, we have the results in Table 1. In Table 2, we show some of the coefficients of the series (14) and the associated Weber number for several values of $\beta$.

Table 1. Some values of coefficients $a_{k}$ for different values of $\alpha$ with $\beta=\frac{\pi}{6}$

\begin{tabular}{|c|c|c|c|c|}
\hline$\alpha$ & $a_{1}$ & $a_{10}$ & $a_{30}$ & $a_{40}$ \\
\hline 15 & $-5,9992 \times 10^{-2}$ & $-1,2431 \times 10^{-5}$ & $-7,4921 \times 10^{-5}$ & $-7,7403 \times 10^{-6}$ \\
\hline 30 & $-2,8782 \times 10^{-2}$ & $1,4646 \times 10^{-4}$ & $1,0553 \times 10^{-5}$ & $7,8078 \times 10^{-7}$ \\
\hline 100 & $-8,4190 \times 10^{-3}$ & $4,8330 \times 10^{-5}$ & $3,0019 \times 10^{-6}$ & $3,4234 \times 10^{-7}$ \\
\hline
\end{tabular}


Table 2. Some values of coefficients $a_{k}$ for different values of $\beta$ with $\alpha=10^{8}$

\begin{tabular}{|c|c|c|c|c|}
\hline$\beta$ & $a_{1}$ & $a_{10}$ & $a_{30}$ & $a_{40}$ \\
\hline$\frac{\pi}{4}$ & $-7,5004 \times 10^{-9}$ & $4,5432 \times 10^{-11}$ & $2,9368 \times 10^{-12}$ & $2,2746 \times 10^{-13}$ \\
\hline$\frac{4 \pi}{6}$ & $-3,3335 \times 10^{-9}$ & $2,0192 \times 10^{-11}$ & $1,3052 \times 10^{-12}$ & $1,0109 \times 10^{-13}$ \\
\hline
\end{tabular}

We can see that there are numerical solutions for the values of the angle $\beta$ between the walls which are shown in Figure 6.
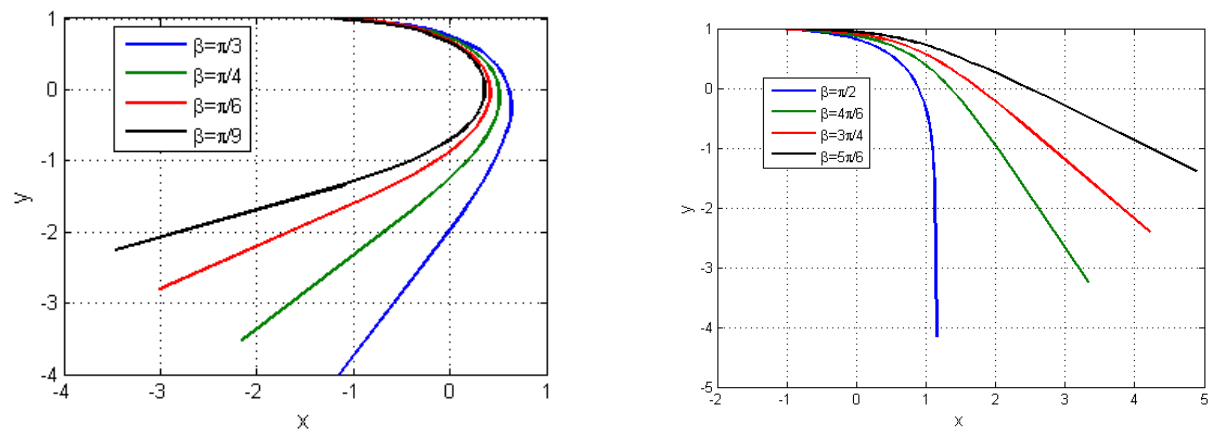

Fig. 6. Free surface shapes for the Weber number $\alpha=10^{8}$ and different values of $\beta$

Furthermore, we see that there are numerical solutions for all $\alpha \geq 15$, while there is no solution for $\alpha \leq 15$. Profiles typical for different Weber numbers of the free surfaces are illustrated in Figure $7 \mathrm{a}$ for $\beta=\frac{\pi}{200}$, and Figure $7 \mathrm{~b}$ for $\beta=\frac{\pi}{4}$.

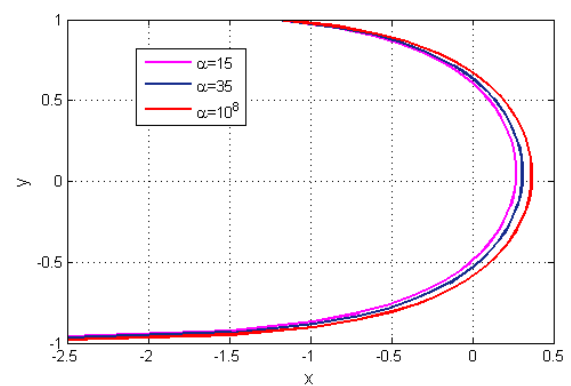

(a) $\beta=\frac{\pi}{200}$

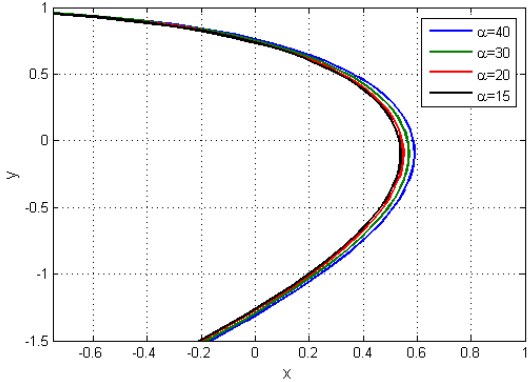

(b) $\beta=\frac{\pi}{4}$

Fig. 7. Free surface shapes for different values of the Weber number $\alpha$

\subsection{The flow without surface tension effect}

In the case where the Weber number trends toward infinity, the effect of surface tension is neglected, so the equation (15) becomes:

$$
e^{2 \bar{\tau}}=1 \quad \text { on the free surface }
$$


In this case, we find the same free surface shape that can be found analytically (see section 4). We have calculated these solutions numerically using the procedure described above, and our results are consistent with the theoretical and experimental results. The comparison of the free current line shapes obtained by our methods with the exact analytical solutions, are presented in Figure 8a, Figure 8b, and Figure 8c for $\beta=0, \beta=\frac{\pi}{4}$, and $\beta=\frac{\pi}{6}$ respectively.

F. Guechi and M. Dahel in [9] study the exact solution of this problem in a special case (when the angle is fixed and equal to 0 ). These results show that they are in good agreement with the results of articles [9] and [10] in a particular case where the angle $\beta$ is equal to 0 and $\frac{\pi}{2}$ respectively.

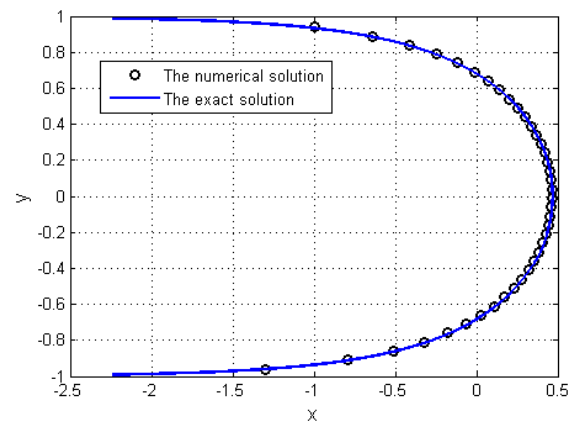

(a) For the Weber number $\alpha=10^{8}$ and $\beta=0$

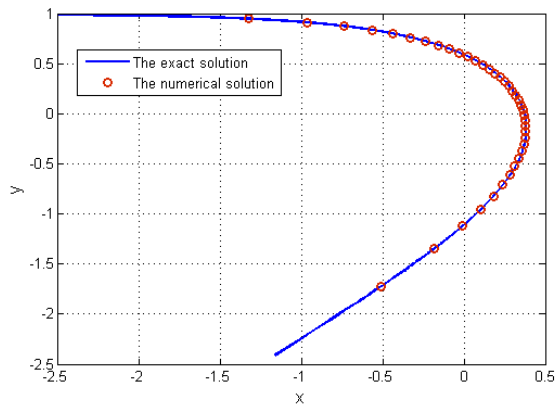

(b) For the Weber number $\alpha=10^{8}$ and $\beta=\frac{\pi}{4}$

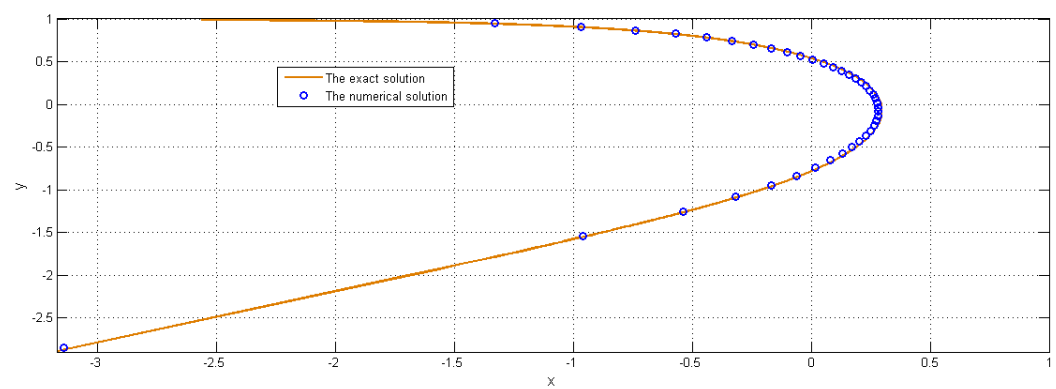

(c) For the Weber number $\alpha=10^{8}$ and $\beta=\frac{\pi}{6}$

Fig. 8. Shape of the free surface flow compared to the exact solution

\section{Conclusion}

This paper is about the problem of a two-dimensional jet with an inviscid and incompressible fluid. This problem is resolved by using two methods. The first is to explicitly and analytically use the hodograph transformation in the case of neglect of the effect of gravity and surface tension. The other one is the numerical use of the Series Truncation Method in case of the effect of surface tension is included, the numerical calculation shows that a solution exists for different values $\alpha \geq 15$. 
If the Weber number large, the obtained results show a good accord between two methods, and the comparison of these surface shapes is illustrated.

\section{Acknowledgments}

The authors would like to express their thanks to the editor and the referees for their helpful comments and suggestions.

\section{References}

[1] Laiadi, A., \& Merzougui, A. (2020). Numerical solution of a cavity problem under surface tension effect. Math. Meth. Appl. Sci., 2020, 1-9.

[2] Panda, S. (2016). A study on inviscid flow with a free surface over an undulating bottom. J. Appl. Fluid Mech., 9(3), 1089-1096.

[3] Panda, S., Martha, S.C., \& Chakrabarti, A. (2016). An alternative approach to study nonlinear inviscid flow over arbitrary bottom topography. Appl. Math. Comput., 273, 165-177.

[4] Panda, S., Martha, S.C., \& Chakrabarti, A. (2015). Three-layer fluid flow over a small obstruction on the bottom of a channel. The ANZIAM Journal, 56(3), 248-274.

[5] Stokes, T.E., Hocking, G.C., \& Forbes, L.K. (2012). Steady free surface flow induced by a submerged ring source or sink. J. Fluid Mech., 694, 352-370.

[6] Chen, M.J., \& Forbes, L.K. (2011). Waves in two-layer shear flow for viscous and inviscid fluids. Eur. J. Mech. B Fluids, 30, 387-404.

[7] Vanden Broeck, J.M., \& Keller, J.B. (1986). Pouring flows. Phys. Fluids, 29, 3958-3961.

[8] Merzougui, A., Mekias, H., \& Guechi, F. (2007). A waveless two-dimensional flow in a channel against an inclined wall with surface tension effect. J. Phys. A: Math. Theor., 40, 14317-14328.

[9] Guechi, F., \& Dahel, M. (2012). Free surface flow modeling between two plates. I. Elec. J. Pure. Appl. Math., 4, 179-184.

[10] Guechi, F., \& Khermache, M. (2012). Free streamline for flow over step. I. J. Pure. Appl. Math., 74, 367-372.

[11] Chapman, S.J., \& Vanden Broeck, J.M. (2002). Exponential asymptotics and capillary waves. Siam J. Appl. Math., 62, 1872-1898.

[12] Choi, J.W. (2002). Free surface waves over a depression. Bull. Austral. Math. Soc., 65, 329-335.

[13] Dias, F., \& Vanden Broeck, J.M. (1989). Open channel flows with submerged obstructions. J. Fluid Mech., 206, 155-170.

[14] Dias, F., \& Vanden Broeck, J.M. (1991). Nonlinear free-surface flow past a submerged inclined flat plate. Phys. Fluids., A3, 2995-3000.

[15] Forbes, L.K., \& Schwartz, L.W. (1982). Free-surface flow over a semicircular obstruction. J. Fluid Mech., 114, 299-314.

[16] Gasmi, A., \& Amara, A. (2018). Free-surface profile of a jet flow in U-shaped channel without gravity effects. Adv. Stud. Contemp. Math. (Kyungshang), 28, 393-400.

[17] Gasmi, A. (2014). Two-dimensional cavitating flow past an oblique plate in a channel. J. Comput. Appl. Math., 259, 828-834.

[18] Ockendon, J.R., Howison, S.D., Lacey, A.A., \& Movichan, A.B. (2003). Applied Partial Differential Equations. Revised Edition Oxford University Press.

[19] Tuck, E.O. (1965). The effect of nonlinearity at the free surface on flow past a submerged cylinder. J. Fluid Mech., 22, 401-414. 
[20] Vanden Broeck, J.M. (1984). Bubbles rising in a tube and jets falling from a nozzle. Phys. Fluids, 27, 1090-1093.

[21] Vanden Broeck, J.M. (1987). Free surface flow over a semi-circular obstruction in a channel. Phys. Fluids, 30, 2315-2317.

[22] Vanden Broeck, J.M. (2010). Gravity-Capillary Free Surface Flows. Cambridge: Cambridge University Press. 